\title{
Theorems on Transform for Product of Generalized M-Series, I-Function of Two Variables and It's Applications
}

Vinita Jain, Ashok Kumar Ronghe, Shailesh Jaloree, and Anil Goyal

\begin{abstract}
In the present paper we establish four theorem which involves I-function of two variables and generalized M-series. In next section we obtain certain new integrals by application of our theorems by giving suitable values to the parameters. Then main theorem reduces to $\mathrm{H}$ function of two variables etc.
\end{abstract}

Keywords - Integral transforms, I-Function, Hypergeometric series, M-series.

\section{INTRODUCTION}

The I-function of two variables represented by means of two contour integrals is defined by Goyal and Agarwal [1] are represented in the following manner :

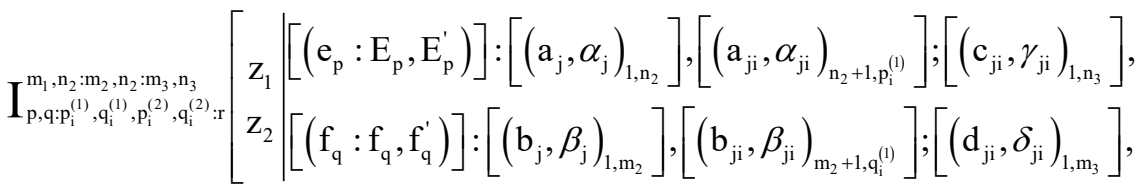

$$
\begin{aligned}
& {\left[\left(\mathrm{c}_{\mathrm{ji}}, \gamma_{\mathrm{ji}}\right)_{\mathrm{n}_{3}+1, \mathrm{p}_{\mathrm{i}}^{(2)}}\right]} \\
& \left.\left[\left(\mathrm{d}_{\mathrm{ji}}, \delta_{\mathrm{ji}}\right)_{\mathrm{m}_{3}+1, \mathrm{q}_{\mathrm{i}}^{(2)}}\right]\right]
\end{aligned}
$$

or

$$
\begin{aligned}
& \mathrm{I}_{\mathrm{p}, \mathrm{q}: \mathrm{QP \textrm {r }}}^{\mathrm{m}, \mathrm{r}, \mathrm{z}}\left[\begin{array}{l|l}
\mathrm{z}_{1} & ((\mathrm{P})),((\mathrm{Q})),((\mathrm{R})),((\mathrm{S})),((\mathrm{T})) \\
\mathrm{z}_{2} & ((\mathrm{U})),((\mathrm{V})),((\mathrm{W})),((\mathrm{X})),((\mathrm{Y}))
\end{array}\right]=\frac{1}{(2 \pi \omega)} \int_{\mathrm{L}_{1} \mathrm{~L}_{2}} \phi_{1}(\xi) \cdot \phi_{2}(\eta) \cdot \psi(\xi \eta) \mathrm{z}_{1}^{\xi} \cdot \mathrm{z}_{2}^{\eta} \mathrm{d} \xi \cdot \mathrm{d} \eta \\
& \phi_{1}(\xi)=\frac{\prod_{j=1}^{\mathrm{m}_{2}} \Gamma\left(\mathrm{b}_{\mathrm{j}}-\beta_{\mathrm{j}} \xi\right) \cdot \prod_{\mathrm{j}=1}^{\mathrm{n}_{2}} \Gamma\left(1-\mathrm{a}_{\mathrm{j}}+\alpha_{\mathrm{j}} \xi\right)}{\sum_{\mathrm{i}=1}^{\mathrm{r}}\left[\prod_{\mathrm{j}=\mathrm{m}_{2}+1}^{\mathrm{q}_{\mathrm{i}}^{(1)}} \Gamma\left(1-\mathrm{b}_{\mathrm{ji}}+\beta_{\mathrm{ji}} \xi\right) \cdot \prod_{\mathrm{j}=\mathrm{n}_{2}+1}^{\mathrm{p}_{\mathrm{i}}^{(1)}} \Gamma\left(\mathrm{a}_{\mathrm{ji}}-\alpha_{\mathrm{ji}} \xi\right)\right]} \\
& \phi_{2}(\eta)=\frac{\prod_{\mathrm{j}=1}^{\mathrm{m}_{3}} \Gamma\left(\mathrm{d}_{\mathrm{j}}-\delta_{\mathrm{j}} \eta\right) \cdot \prod_{\mathrm{j}=1}^{\mathrm{n}_{3}} \Gamma\left(1-\mathrm{c}_{\mathrm{j}}+\gamma_{\mathrm{j}} \eta\right)}{\sum_{\mathrm{i}=1}^{\mathrm{r}}\left[\prod_{\mathrm{j}=\mathrm{m}_{2}+1}^{\mathrm{q}_{\mathrm{i}}^{(2)}} \Gamma\left(1-\mathrm{d}_{\mathrm{ji}}+\delta_{\mathrm{ji}} \eta\right) \cdot \prod_{\mathrm{j}=\mathrm{n}_{2}+1}^{\mathrm{p}_{\mathrm{i}}^{(2)}} \Gamma\left(\mathrm{c}_{\mathrm{ji}}-\gamma_{\mathrm{ji}} \eta\right)\right]}
\end{aligned}
$$

Published on December 13, 2021.

V. Jain, Department of Pure \& Applied Mathematics, S.S.L. Jain P.G. College, Vidisha (M.P.), India. (corresponding e-mail: vinita08.82@gmail.com)

A. K. Ronghe, Department of Pure \& Applied Mathematics, S.S.L. Jain P.G. College, Vidisha (M.P.), India.

S. Jaloree, Department of Applied Mathematics \& Computer Science, S.A.T.I., Vidisha (M.P.), India.

A. Goyal, Department of Applied Mathematics, R.G.P.V., Bhopal (M.P.), India. 


$$
\psi(\xi, \eta)=\frac{\prod_{\mathrm{j}=1}^{\mathrm{m}_{1}} \Gamma\left(\mathrm{f}_{\mathrm{j}}-\mathrm{F}_{\mathrm{j}} \xi-\mathrm{F}_{\mathrm{j}} \eta\right) \cdot \prod_{\mathrm{j}=1}^{\mathrm{n}_{1}} \Gamma\left(1-\mathrm{e}_{\mathrm{j}}+\mathrm{E}_{\mathrm{j}} \xi+\mathrm{E}_{\mathrm{j}} \eta\right)}{\prod_{\mathrm{j}=\mathrm{m}_{1}+1}^{\mathrm{q}} \Gamma\left(1-\mathrm{f}_{\mathrm{j}} \xi+\mathrm{F}_{\mathrm{j}} \eta\right) \cdot \prod_{\mathrm{j}=\mathrm{n}_{1}+1}^{\mathrm{p}} \Gamma\left(\mathrm{e}_{\mathrm{j}}-\mathrm{E}_{\mathrm{j}} \xi-\mathrm{E}_{\mathrm{j}} \eta\right)}
$$

$\mathrm{z}_{1}$ and $\mathrm{z}_{2}$ are two non zero complex variables. $\mathrm{L}_{1}$ and $\mathrm{L}_{2}$ are double Mellin-Barnes type contour integrals and convergence condition are as follow :

where,

$$
\left|\arg \left(\mathrm{z}_{1}\right)<\frac{\pi \mathrm{Ai}}{2}, \quad\right| \arg \left(\mathrm{z}_{2}\right)<\frac{\pi \mathrm{Bi}}{2}
$$

$$
\begin{aligned}
& A i=\sum_{j=1}^{n_{1}} E_{j}-\sum_{j=n_{1}+1}^{p} E_{j}+\sum_{j=1}^{m_{1}} F_{j}-\sum_{j=m_{1}+1}^{q} F_{j}+\sum_{j=1}^{m_{2}} \beta_{j}-\sum_{j=m_{2}+1}^{q^{(1)}} \beta_{j}+\sum_{j=1}^{m_{2}} \alpha_{j}-\sum_{j=n_{2}+1}^{q^{(2)}} \alpha_{j}>0 \\
& B i=\sum_{j=1}^{n_{1}} E_{j}^{\prime}-\sum_{j=n_{1}+1}^{p} E_{j}+\sum_{j=1}^{m_{1}} F_{j}^{\prime}-\sum_{j=m_{1}+1}^{q} F_{j}^{\prime}+\sum_{j=1}^{m_{3}} \delta_{j}-\sum_{j=m_{3}+1}^{q_{i}^{(2)}} \delta_{j}+\sum_{j=1}^{n_{3}} \gamma_{j}-\sum_{j=n_{3}+1}^{p_{1}^{(2)}} \gamma_{j}>0
\end{aligned}
$$

where $\mathrm{m}_{1}, \mathrm{n}_{1} ; \mathrm{m}_{2}, \mathrm{n}_{2} ; \mathrm{m}_{3}, \mathrm{n}_{3}$ and $\mathrm{p}, \mathrm{q}: \mathrm{p}_{\mathrm{i}}^{(1)}, \mathrm{q}_{\mathrm{i}}^{(1)}: \mathrm{p}_{\mathrm{i}}^{(2)}, \mathrm{q}_{\mathrm{i}}^{(2)}$ are non zero integers satisfying the conditions.

$$
0 \leq \mathrm{n}_{1} \leq \mathrm{p}, 0 \leq \mathrm{n}_{2} \leq \mathrm{p}_{\mathrm{i}}^{(1)}, 0 \leq \mathrm{n}_{3} \leq \mathrm{p}_{\mathrm{i}}^{(2)}, 0 \leq \mathrm{m}_{1} \leq \mathrm{q}, 0 \leq \mathrm{m}_{2} \leq \mathrm{q}_{\mathrm{i}}^{(1)}, 0 \leq \mathrm{m}_{3} \leq \mathrm{q}_{\mathrm{i}}^{(2)} \text { you all } \mathrm{i}=1,2, \ldots, \mathrm{r},
$$
where $r$ is positive integers, for asymptotic expansion and all other conditions, (see [1]). These condition will be assumed two hold good in the I-function of two variables occurring in this paper.

The generalized M-series is defined as:

$$
{ }_{\mathrm{p}} \mathbf{M}_{\mathrm{q}}^{\alpha, \beta}(\mathrm{z})={ }_{\mathrm{p}} \mathbf{M}_{1}^{\alpha, \beta}\left(\mathrm{a}_{1} \ldots \ldots \mathrm{a}_{\mathrm{p}} ; \mathrm{b}_{1} \ldots \ldots \mathrm{b}_{\mathrm{q}} ; \mathrm{z}\right)=\sum_{\mathrm{k}=0}^{\infty} \frac{\left(\mathrm{a}_{1}\right)_{\mathrm{k}} \ldots . .\left(\mathrm{a}_{\mathrm{p}}\right)_{\mathrm{k}}}{\left(\mathrm{b}_{1}\right)_{\mathrm{k}} \ldots . .\left(\mathrm{b}_{\mathrm{q}}\right)_{\mathrm{k}}} \cdot \frac{\mathrm{zk}}{\Gamma(\alpha \mathrm{k}+\beta)}
$$

where $\alpha, \beta \in \mathrm{c}, \mathrm{z} \in \mathrm{c}, \operatorname{Re}(\alpha)>0 ;\left(\mathrm{q}_{\mathrm{i}}\right)_{\mathrm{k}}(\mathrm{i}=1, \ldots ., \mathrm{p})$ and $\left(\mathrm{b}_{\mathrm{j}}\right)_{\mathrm{k}}(\mathrm{i}=1, \ldots ., \mathrm{q})$ are Pochhammer symbols. The series (8) is defined when none of the parameters $\left(b_{j}\right)_{k}$ is a negative $a_{i}$ is a negative integer or zero, then series terminates to a polynomial in $\mathrm{z}$. The series (8) is convergent for all $\mathrm{z}$ if $\mathrm{p} \leq \mathrm{q}$; it is convergent for $|\mathrm{z}|<\delta=\alpha^{\alpha}$ if $\mathrm{p}=\mathrm{q}+1$ and divergent if $\mathrm{p}>\mathrm{q}+1$. When $\mathrm{p}=\mathrm{q}+1$ and $|\mathrm{z}|<\delta$, the series is convergent on conditions depending on the parameters. The detailed account of the M-series are given in [2].

II. THEOREM-1

$$
\begin{aligned}
& \int_{0}^{1} \mathrm{z}^{\mathrm{p}-1},(\mathrm{t}-\mathrm{z})^{\sigma-1},{ }_{\mathrm{p}} \mathrm{M}_{\mathrm{q}}^{\alpha, \beta}\left[\mathrm{bz}^{v}(\mathrm{t}-\mathrm{z})^{\tau}\right] \cdot \mathrm{I}_{\mathrm{p}, \mathrm{m}, \mathrm{p}: \mathrm{p}: \mathrm{r}}\left[\begin{array}{c|c}
\omega_{1} \mathrm{Z}^{\mathrm{v}_{1}}(\mathrm{t}-\mathrm{z})^{\tau_{1}} & ((\mathrm{P})),((\mathrm{Q})),((\mathrm{R})),((\mathrm{S})),((\mathrm{T})) \\
\omega_{2} \mathrm{z}^{\nu_{2}}(\mathrm{t}-\mathrm{z})^{\tau_{2}} & ((\mathrm{U})),((\mathrm{V})),((\mathrm{W})),((\mathrm{X})),((\mathrm{Y}))
\end{array}\right] \mathrm{dz} \\
& =\mathrm{t}^{\sigma+\mathrm{p}-\mathrm{z}} \sum_{\mathrm{k}=0}^{\infty} \frac{\left(\mathrm{a}_{1}\right)_{\mathrm{k}} \ldots . .\left(\mathrm{a}_{\mathrm{p}}\right)_{\mathrm{k}}}{\left(\mathrm{b}_{1}\right)_{\mathrm{k}} \ldots . .\left(\mathrm{b}_{\mathrm{q}}\right)_{\mathrm{k}}}, \frac{\left[\mathrm{bt}^{v+\tau}\right]^{\mathrm{k}}}{\Gamma(\alpha \mathrm{k}+\beta)} . \\
& \mathrm{I}_{\mathrm{p}_{1}+2, \mathrm{q}_{1}+1: \mathrm{P}: \mathrm{r}}^{\mathrm{m}, \mathrm{r}+;}\left[\begin{array}{c|c}
\omega_{1} \mathrm{t}^{\left(\mathrm{v}_{1}+\tau\right) \cdot \mathrm{z}} & \left(\left(\mathrm{X}_{1}\right)\right),((\mathrm{P})),((\mathrm{Q})),((\mathrm{S})),((\mathrm{T})) \\
\omega_{2} \mathrm{t}^{\left(\mathrm{v}_{2}+\tau\right) \cdot \mathrm{z}} & ((\mathrm{U})),\left(\left(\mathrm{X}_{2}\right)\right),((\mathrm{V})),((\mathrm{W})),((\mathrm{X})),((\mathrm{Y}))
\end{array}\right]
\end{aligned}
$$

where $\mathrm{X}_{1}$ and $\mathrm{X}_{2}$ are as follows:

$$
\mathrm{X}_{1} \equiv\left\{\left(+1-\mathrm{p}-v \mathrm{k}: v_{1}, v_{2}\right),\left(+1-\sigma-\tau \mathrm{k}: \tau_{1}, \tau_{2}\right)\right\}
$$




$$
\mathrm{X}_{2} \equiv\left\{(+1-\sigma--\mathrm{p}-v \mathrm{k}-\tau \mathrm{k}):\left(v_{1}+\tau_{1}\right),\left(v_{2}+\tau_{2}\right)\right\}
$$

provided that,

(i) $\alpha>0, \beta>0$

(ii) $\operatorname{Re}\left[\mathrm{p}+\tau_{1}\left[\min _{1<\mathrm{j}<\mathrm{m}_{2}}\left(\mathrm{~b}_{\mathrm{j}} / \beta_{\mathrm{j}}\right)\right]+\tau_{2}\left[\min _{1<\mathrm{j}<\mathrm{m}_{3}}\left(\mathrm{c}_{\mathrm{j}} / \gamma_{\mathrm{j}}\right)\right]\right]>0$,

(iii) $\operatorname{Re}\left[\mathrm{p}+v_{1}\left[\min _{1<\mathrm{j}<\mathrm{m}_{2}}\left(\mathrm{~b}_{\mathrm{j}} / \beta_{\mathrm{j}}\right)\right]+v_{2}\left[\min _{1<\mathrm{j}<\mathrm{m}_{3}}\left(\mathrm{c}_{\mathrm{j}} / \gamma_{\mathrm{j}}\right)\right]\right]>0$,

(iv) $\quad \Delta_{1}<\frac{1}{2} \pi \mathrm{Ai}, \Delta_{2}<\frac{1}{2} \pi \mathrm{Bi}, \forall \mathrm{i} \in(1,2, \ldots \ldots, \mathrm{r})$,

where Ai and Bi are mentioned in (6) and (7) respectively.

\section{THEOREM-2}

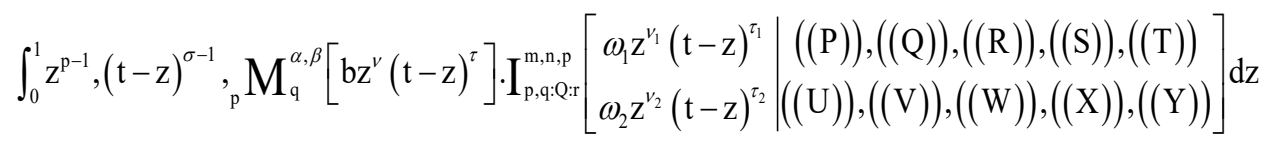

$$
\begin{aligned}
& =\mathrm{t}^{\sigma+\mathrm{p}-\mathrm{z}} \sum_{\mathrm{k}=0}^{\infty} \frac{\left(\mathrm{a}_{1}\right)_{\mathrm{k}} \ldots . .\left(\mathrm{a}_{\mathrm{p}}\right)_{\mathrm{k}}}{\left(\mathrm{b}_{1}\right)_{\mathrm{k}} \ldots . .\left(\mathrm{b}_{\mathrm{q}}\right)_{\mathrm{k}}}, \frac{\left[\mathrm{bt}^{v+\tau}\right]^{\mathrm{k}}}{\Gamma(\alpha \mathrm{k}+\beta)} \text {. }
\end{aligned}
$$

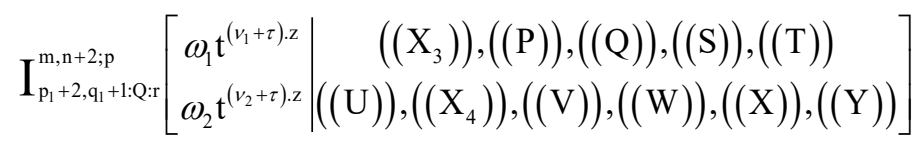

where $\mathrm{X}_{1}$ and $\mathrm{X}_{2}$ are as follows:

$$
\begin{gathered}
\mathrm{X}_{1} \equiv\left\{\left(+1-\mathrm{p}-v \mathrm{k}: v_{1}, v_{2}\right),\left(+1-\sigma-\tau \mathrm{k}: \tau_{1}, \tau_{2}\right)\right\} \\
\mathrm{X}_{2} \equiv\left\{(+1-\sigma--\mathrm{p}-v \mathrm{k}-\tau \mathrm{k}):\left(v_{1}+\tau_{1}\right),\left(v_{2}+\tau_{2}\right)\right\}
\end{gathered}
$$

provided that:

(i) $\quad \alpha>0, \beta>0$,

(ii) $\operatorname{Re}\left[\mathrm{p}+\tau_{1}\left[\min _{1<\mathrm{j}<\mathrm{m}_{2}}\left(\mathrm{~b}_{\mathrm{j}} / \beta_{\mathrm{j}}\right)\right]+\tau_{2}\left[\min _{1<\mathrm{j}<\mathrm{m}_{3}}\left(\mathrm{c}_{\mathrm{j}} / \gamma_{\mathrm{j}}\right)\right]\right]>0$,

(iii) $\operatorname{Re}\left[\mathrm{p}+v_{1}\left[\min _{1<\mathrm{j}<\mathrm{m}_{2}}\left(\mathrm{~b}_{\mathrm{j}} / \beta_{\mathrm{j}}\right)\right]+v_{2}\left[\min _{1<\mathrm{j}<\mathrm{m}_{3}}\left(\mathrm{c}_{\mathrm{j}} / \gamma_{\mathrm{j}}\right)\right]\right]>0$,

(iv) $\Delta_{1}<\frac{1}{2} \pi \mathrm{Ai}, \Delta_{2}<\frac{1}{2} \pi \mathrm{Bi}, \forall \mathrm{i} \in(1,2, \ldots . ., \mathrm{r})$,

where $\mathrm{Ai}$ and $\mathrm{Bi}$ are mentioned in (6) and (7) respectively.

\section{HEOREM-3}

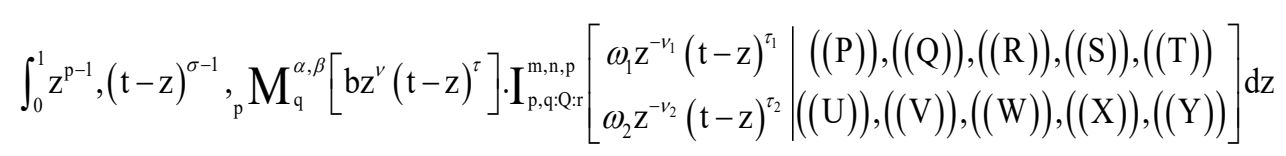




$$
\begin{gathered}
=\mathrm{t}^{\sigma+\mathrm{p}-\mathrm{z}} \sum_{\mathrm{k}=0}^{\infty} \frac{\left(\mathrm{a}_{1}\right)_{\mathrm{k}} \ldots \ldots .\left(\mathrm{a}_{\mathrm{p}}\right)_{\mathrm{k}}}{\left(\mathrm{b}_{1}\right)_{\mathrm{k}} \ldots \ldots .\left(\mathrm{b}_{\mathrm{q}}\right)_{\mathrm{k}}}, \frac{\left[\mathrm{bt}^{(v+\tau)}\right]^{\mathrm{k}}}{\Gamma(\alpha \mathrm{k}+\beta)} . \\
\mathrm{I}_{\mathrm{p}_{1}+1, \mathrm{q}+2: \mathrm{Q}: \mathrm{r}}^{\mathrm{m}+\mathrm{n}+\mathrm{r}+\mathrm{p}}\left[\begin{array}{c|c}
\omega_{1} \mathrm{t}^{\left(\tau_{1}-v_{1}\right) \cdot z} & \left(\left(\mathrm{X}_{5}\right)\right),((\mathrm{P})),((\mathrm{Q})),((\mathrm{S})),((\mathrm{T})) \\
\omega_{2} \mathrm{t}^{\left(\tau_{2}-v_{2}\right) \cdot \mathrm{z}} & ((\mathrm{U})),\left(\left(\mathrm{X}_{6}\right)\right),((\mathrm{V})),((\mathrm{W})),((\mathrm{X})),((\mathrm{Y}))
\end{array}\right]
\end{gathered}
$$

where $((\mathrm{P})),((\mathrm{Q})),((\mathrm{R})),((\mathrm{S})),((\mathrm{T})),((\mathrm{U})),((\mathrm{V})),((\mathrm{W})),((\mathrm{X})),((\mathrm{Y}))$ are sets of parameters given in (1) and $\left(\left(\mathrm{X}_{5}\right)\right),\left(\left(\mathrm{X}_{6}\right)\right)$ are as follows:

$$
\begin{gathered}
\mathrm{X}_{5} \equiv\left\{\left(\mathrm{p}-v \mathrm{k}: v_{1}, v_{2}\right),\left(1-\sigma-\tau \mathrm{k}: \tau_{1}, \tau_{2}\right)\right\} \\
\mathrm{X}_{6} \equiv\left\{(1-\mathrm{p}-\sigma-v \mathrm{k}-\tau \mathrm{k}):\left(\tau_{1}-v_{1}\right),\left(\tau_{2}-v_{2}\right)\right\}
\end{gathered}
$$

provided that

(i) $\alpha>0, \beta>0$,

(ii) $\operatorname{Re}\left[\sigma+\tau_{1}\left[\min _{1<\mathrm{j}<\mathrm{m}_{2}}\left(\mathrm{~b}_{\mathrm{j}} / \beta_{\mathrm{j}}\right)\right]+\tau_{2}\left[\min _{1<\mathrm{j}<\mathrm{m}_{3}}\left(\mathrm{c}_{\mathrm{j}} / \gamma_{\mathrm{j}}\right)\right]\right]>0$,

(iii) $\operatorname{Re}\left[\mathrm{p}+v_{1}\left[\min _{1<\mathrm{j}<\mathrm{m}_{2}}\left(\mathrm{~b}_{\mathrm{j}} / \beta_{\mathrm{j}}\right)\right]+v_{2}\left[\min _{1<\mathrm{j}<\mathrm{m}_{3}}\left(\mathrm{c}_{\mathrm{j}} / \gamma_{\mathrm{j}}\right)\right]\right]>0$,

(iv) $\Delta_{1}<\frac{1}{2} \pi \mathrm{Ai}, \Delta_{2}<\frac{1}{2} \pi \mathrm{Bi}, \forall \mathrm{i} \in(1,2, \ldots . ., \mathrm{r})$,

where $\mathrm{Ai}$ and $\mathrm{Bi}$ are mentioned in (6) and (7) respectively.

\section{THEOREM-4}

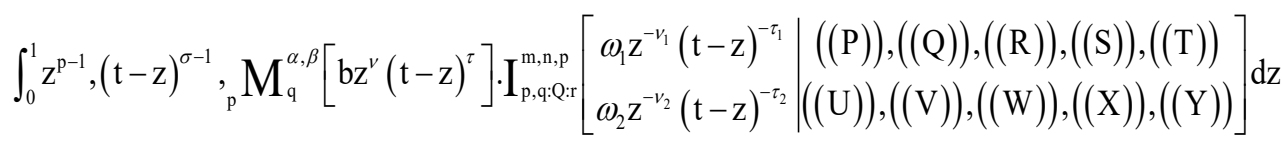

$$
\begin{aligned}
& =\mathrm{t}^{\sigma+\mathrm{p}-2} \sum_{\mathrm{k}=0}^{\infty} \frac{\left(\mathrm{a}_{1}\right)_{\mathrm{k}} \ldots . .\left(\mathrm{a}_{\mathrm{p}}\right)_{\mathrm{k}}}{\left(\mathrm{b}_{1}\right)_{\mathrm{k}} \ldots . .\left(\mathrm{b}_{\mathrm{q}}\right)_{\mathrm{k}}}, \frac{\left[\mathrm{bt}^{(\nu+\tau)}\right]^{\mathrm{k}}}{\Gamma(\alpha \mathrm{k}+\beta)} .
\end{aligned}
$$

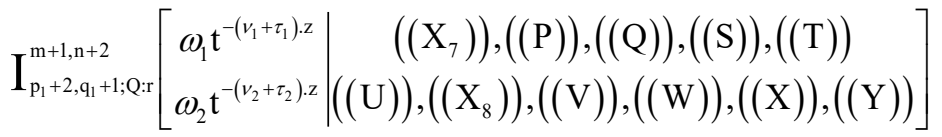

where $((\mathrm{P})),((\mathrm{Q})),((\mathrm{R})),((\mathrm{S})),((\mathrm{T})),((\mathrm{U})),((\mathrm{V})),((\mathrm{W})),((\mathrm{X})),((\mathrm{Y}))$ are sets of parameters given in (1) and $\left(\left(\mathrm{X}_{7}\right)\right),\left(\left(\mathrm{X}_{8}\right)\right)$ are as follows:

$$
\begin{gathered}
\mathrm{X}_{7} \equiv\left\{\left(\mathrm{p}+v \mathrm{k}: v_{1}, v_{2}\right),\left(\sigma+\tau \mathrm{k}: \tau_{1}, \tau_{2}\right)\right\}, \\
\mathrm{X}_{8} \equiv\left\{\mathrm{p}+\sigma+(v+\tau) \mathrm{k} ;\left(v_{1}+v_{2}\right),\left(\tau_{1}+\tau_{2}\right)\right\},
\end{gathered}
$$

provided that:

(i) $\alpha>0, \beta>0$,

(ii) $\operatorname{Re}\left[\sigma+\tau_{1}\left[\min _{1<\mathrm{j}<\mathrm{m}_{2}}\left(\mathrm{~b}_{\mathrm{j}} / \beta_{\mathrm{j}}\right)\right]+\tau_{2}\left[\min _{1<\mathrm{j}<\mathrm{m}_{3}}\left(\mathrm{c}_{\mathrm{j}} / \gamma_{\mathrm{j}}\right)\right]\right]>0$,

(iii) $\operatorname{Re}\left[\mathrm{p}+v_{1}\left[\min _{1<\mathrm{j}<\mathrm{m}_{2}}\left(\mathrm{~b}_{\mathrm{j}} / \beta_{\mathrm{j}}\right)\right]+v_{2}\left[\min _{1<\mathrm{j}<\mathrm{m}_{3}}\left(\mathrm{c}_{\mathrm{j}} / \gamma_{\mathrm{j}}\right)\right]\right]>0$, 
(iv) $\Delta_{1}<\frac{1}{2} \pi \mathrm{Ai}, \Delta_{2}<\frac{1}{2} \pi \mathrm{Bi}, \forall \mathrm{i} \in(1,2, \ldots ., \mathrm{r})$

where $\mathrm{Ai}$ and $\mathrm{Bi}$ are mentioned in (6) and (7) respectively.

\section{PROOF}

If we apply the definition of I-function of two variables and M-series to the given (9) integral, we have:

$$
\begin{aligned}
& \int_{0}^{\mathrm{t}} \mathrm{z}^{\mathrm{p}-1},(\mathrm{t}-\mathrm{z})^{\sigma-1}, \mathrm{p}_{\mathrm{p}}^{\alpha, \beta}\left[\mathrm{bz}^{v}(\mathrm{t}-\mathrm{z})^{\tau}\right] \cdot \mathrm{I}_{\mathrm{p}, \mathrm{q}: \mathrm{Q}: \mathrm{r}}^{\mathrm{m}, \mathrm{n}, \mathrm{p}}\left[\begin{array}{c|c}
\omega_{1} \mathrm{z}^{v_{1}}(\tau-\mathrm{z})^{\tau_{1}} & ((\mathrm{P})),((\mathrm{Q})),((\mathrm{R})),((\mathrm{S})),((\mathrm{T})) \\
\omega_{2} \mathrm{z}^{\nu_{2}}(\tau-\mathrm{z})^{\tau_{2}} & ((\mathrm{U})),((\mathrm{V})),((\mathrm{W})),((\mathrm{X})),((\mathrm{Y}))
\end{array}\right] \mathrm{dz} \\
& \int_{0}^{\mathrm{t}} \mathrm{z}^{\mathrm{p}-1},(\mathrm{t}-\mathrm{z})^{\sigma-1}, \sum_{\mathrm{k}=0}^{\infty} \frac{\left(\mathrm{a}_{1}\right)_{\mathrm{k}} \cdots \cdots\left(\mathrm{a}_{\mathrm{p}}\right)_{\mathrm{k}}}{\left(\mathrm{b}_{1}\right)_{\mathrm{k}} \ldots \ldots\left(\mathrm{b}_{\mathrm{q}}\right)_{\mathrm{k}}}, \frac{\mathrm{b}^{\mathrm{k}} \mathrm{z}^{\nu \mathrm{k}}(\mathrm{t}-\mathrm{z})^{\tau \mathrm{k}}}{\Gamma(\alpha \mathrm{k}+\beta)} \\
& \times \frac{1}{(2 \pi \omega)^{2}} \int_{\mathrm{L}_{1}} \int_{\mathrm{L}_{2}} \phi_{1}(\xi) \cdot \phi_{2}(\eta) \cdot \psi(\xi, \eta) \omega_{1}^{\xi} \mathrm{z}^{\nu_{1} \xi}(\mathrm{t}-\mathrm{z})^{\tau_{1} \xi} \cdot \omega_{2}^{\eta} \mathrm{z}^{\nu_{2} \eta}(\mathrm{t}-\mathrm{z})^{\tau_{2} \eta} \mathrm{d} \xi \cdot \mathrm{d} \eta \cdot \mathrm{dz}
\end{aligned}
$$

Interchanging the order of integration and summation which is permissible under the condition stated with theorem, we get:

$$
\begin{aligned}
& \sum_{\mathrm{k}=0}^{\infty} \frac{\left(\mathrm{a}_{1}\right)_{\mathrm{k}} \cdots \cdots\left(\mathrm{a}_{\mathrm{p}}\right)_{\mathrm{k}}}{\left(\mathrm{b}_{1}\right)_{\mathrm{k}} \ldots \ldots\left(\mathrm{b}_{\mathrm{q}}\right)_{\mathrm{k}}}, \frac{\mathrm{b}^{\mathrm{k}} \mathrm{t}^{(\nu \tau)}}{\Gamma(\alpha \mathrm{k}+\beta)} \cdot \int_{0}^{\mathrm{t}} \mathrm{z}^{\mathrm{p}+v \mathrm{k}+v_{1} \xi+v_{2} \eta} \cdot(\mathrm{t}-\mathrm{z})^{\sigma+\tau \mathrm{k}+\tau_{1} \xi+\tau_{2} \eta-1} \\
& \times \frac{1}{(2 \pi \omega)^{2}} \int_{\mathrm{L}_{1}} \int_{\mathrm{L}_{2}} \phi_{1}(\xi) \cdot \phi_{2}(\eta) \cdot \psi(\xi, \eta) \omega_{1}^{\xi} \mathrm{d} \xi \cdot \omega_{2}^{\eta} \mathrm{d} \xi \cdot \mathrm{d} \eta \cdot \mathrm{dz}
\end{aligned}
$$

Substituting $\mathrm{z}=\mathrm{st}$ and, using the formula of Beta function and after adjusting the terms we get theorem (9).

\section{APPLICATIONS}

(i) If we set $v_{1}=0$ and $v_{2}=0$ in (9) then we get,

$$
\begin{aligned}
& \int_{0}^{\mathrm{t}} \mathrm{z}^{\mathrm{p}-1},(\mathrm{t}-\mathrm{z})^{\sigma-1}, \mathrm{M}_{\mathrm{p}}^{\alpha, \beta}\left[\mathrm{bz}^{v}(\mathrm{t}-\mathrm{z})^{\tau}\right] \cdot \mathrm{I}_{\mathrm{p}, \mathrm{q}: \mathrm{Q}: \mathrm{r}}^{\mathrm{m}, \mathrm{n},}\left[\begin{array}{c|c}
\omega_{1}(\tau-\mathrm{z})^{\tau_{1}} & ((\mathrm{P})),((\mathrm{Q})),((\mathrm{R})),((\mathrm{S})),((\mathrm{T})) \\
\omega_{2}(\tau-\mathrm{z})^{\tau_{2}} & ((\mathrm{U})),((\mathrm{V})),((\mathrm{W})),((\mathrm{X})),((\mathrm{Y}))
\end{array}\right] \mathrm{dz} \\
& \mathrm{t}^{\sigma+\mathrm{p}-2} \cdot \sum_{\mathrm{k}=0}^{\infty} \frac{\left(\mathrm{a}_{1}\right)_{\mathrm{k}} \cdots \cdots\left(\mathrm{a}_{\mathrm{p}}\right)_{\mathrm{k}}}{\left(\mathrm{b}_{1}\right)_{\mathrm{k}} \ldots \ldots\left(\mathrm{b}_{\mathrm{q}}\right)_{\mathrm{k}}}, \frac{\left(\mathrm{bt}^{v+\tau}\right)^{\mathrm{k}}}{\Gamma(\alpha \mathrm{k}+\beta)} \cdot \Gamma(\mathrm{p}+v \mathrm{k}) \\
& \mathrm{I}_{\mathrm{p}+1, \mathrm{q}+1: \mathrm{Q}: \mathrm{r}}^{\mathrm{m}, \mathrm{r}, \mathrm{r}}\left[\begin{array}{c|c}
\omega_{1} \mathrm{t}^{\tau_{1}} \mathrm{z} & \left(\left(\mathrm{x}_{9}\right)\right),((\mathrm{P})),((\mathrm{Q})),((\mathrm{R})),((\mathrm{S})),((\mathrm{T})) \\
\omega_{2} \mathrm{t}^{\tau_{2}} \mathrm{z} & ((\mathrm{U})),\left(\left(\mathrm{x}_{10}\right)\right),((\mathrm{V})),((\mathrm{Q})),((\mathrm{X})),((\mathrm{Y}))
\end{array}\right]
\end{aligned}
$$

where,

$$
\begin{gathered}
\mathrm{X}_{9} \equiv\left\{\left(1-\sigma-\tau \mathrm{k}: \tau_{1}, \tau_{2}\right)\right\}, \\
\mathrm{X}_{10} \equiv\left\{\left(1-\sigma-\mathrm{p}-\nu \mathrm{k}-\tau \mathrm{k}: \tau_{1}, \tau_{2}\right)\right\},
\end{gathered}
$$

provided that:

(1) $\alpha, \beta>0$,

(2) $\operatorname{Re}\left[\sigma+\tau_{1}\left[\min _{1<\mathrm{j}<\mathrm{m}_{2}}\left(\mathrm{~b}_{\mathrm{j}} / \beta_{\mathrm{j}}\right)\right]+\tau_{2}\left[\min _{1<\mathrm{j}<\mathrm{m}_{3}}\left(\mathrm{c}_{\mathrm{j}} / \gamma_{\mathrm{j}}\right)\right]\right]>0$, 


$$
\Delta_{1}<\frac{1}{2} \pi \mathrm{Ai}, \Delta_{2}<\frac{1}{2} \pi \mathrm{Bi}, \forall \mathrm{i} \in(1,2, \ldots, \mathrm{r}),
$$

where $\mathrm{Ai}$ and $\mathrm{Bi}$ are mentioned in (6) and (7) respectively.

(ii) If we set $\tau_{1}=0$ and $\tau_{2}=0$ in (9) then we get:

$$
\begin{aligned}
& \int_{0}^{\mathrm{t}} \mathrm{z}^{\mathrm{p}-1},(\mathrm{t}-\mathrm{z})^{\sigma-1},{ }_{\mathrm{p}} \mathrm{M}_{\mathrm{q}}^{\alpha, \beta}\left[\mathrm{bz}^{v}(\mathrm{t}-\mathrm{z})^{\tau}\right] \cdot \mathrm{I}_{\mathrm{p}, \mathrm{q}: \mathrm{Q}: \mathrm{r}}^{\mathrm{m}, \mathrm{p},[}\left[\begin{array}{c|c}
\omega_{1} \mathrm{z}^{\mathrm{v}_{1}} & ((\mathrm{P})),((\mathrm{Q})),((\mathrm{R})),((\mathrm{S})),((\mathrm{T})) \\
\omega_{2} \mathrm{z}^{\mathrm{v}_{2}} & ((\mathrm{U})),((\mathrm{V})),((\mathrm{W})),((\mathrm{X})),((\mathrm{Y}))
\end{array}\right] \\
& \mathrm{t}^{\sigma+\mathrm{p}-2} \cdot \sum_{\mathrm{k}=0}^{\infty} \frac{\left(\mathrm{a}_{1}\right)_{\mathrm{k}} \ldots . .\left(\mathrm{a}_{\mathrm{p}}\right)_{\mathrm{k}}}{\left(\mathrm{b}_{1}\right)_{\mathrm{k}} \ldots . .\left(\mathrm{b}_{\mathrm{q}}\right)_{\mathrm{k}}}, \frac{\left(\mathrm{bt}^{v+\tau}\right)^{\mathrm{k}}}{\Gamma(\alpha \mathrm{k}+\beta)} \cdot \Gamma(\sigma+\tau \mathrm{k}) .
\end{aligned}
$$

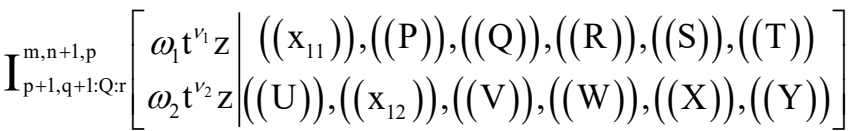

where,

$$
\begin{gathered}
\mathrm{X}_{11} \equiv\left\{(1-\mathrm{p}-v \mathrm{k}): v_{1}, v_{2}\right\}, \\
\mathrm{X}_{12} \equiv\left\{(1-\mathrm{p}-\sigma-v \mathrm{k}-\tau \mathrm{k}): v_{1}, v_{2}\right\},
\end{gathered}
$$

provided that

(1) $\alpha, \beta>0$,

(2) $\operatorname{Re}\left\{\mathrm{p}+v_{1}\left[\min _{1<\mathrm{j}<\mathrm{m}_{2}}\left(\mathrm{~b}_{\mathrm{j}} / \beta_{\mathrm{j}}\right)\right]+v_{2}\left[\min _{1<\mathrm{j}<\mathrm{m}_{3}}\left(\mathrm{c}_{\mathrm{j}} / \gamma_{\mathrm{j}}\right)\right]\right\}>0$,

(3) $\Delta_{1}<\frac{1}{2} \pi \mathrm{Ai}, \Delta_{2}<\frac{1}{2} \pi \mathrm{Bi}$,

where $\mathrm{Ai}$ and $\mathrm{Bi}$ are mentioned in (6) and (7) respectively.

\section{CONFLICT OF INTEREST}

Authors declare that they do not have any conflict of interest.

\section{REFERENCES}

[1] Goyal A, Agrawal RD. Fourier series for I-function of two variables, Proc. Annu. Conf. Banaras Math. Sec. Varanasi Dec., 1994; 10-12.

[2] Sharma M, Jain R. A note on a generalized M-series as a special function of fractional calculus. Fract. Calc. Appl. Anal. 2009; 12(4): 449-52.

[3] Naderi SH, Bestwick JP, Wald DS. Adherence to drugs that prevent cardiovascular disease: meta-analysis on 376,162 patients. Am J Med. 2012; 125(9): 882-887.

[4] Goyal A, Agrawal RD. Integral involving the product of I-function of two variables. Journal of MACT. 1995; $28: 147-55$.

[5] Rainville ED. Special Functions, Chelsea Publ. Co., Bronx, New York. 1971.

[6] Chouhan A, Khan AM. Unified integrals associated with H-functions and M-series. J. Frac. Cal. Appl. 2015; 6(2): 11-7.

[7] Srivastava HM, Gupta KC, Goyal SP. The H-functions of one and two variables, with applications. South Asian Publishers; 1982.

[8] Mathai AM, Saxena RK, Haubold HJ. The H-function: theory and applications. Springer Science \& Business Media; 2009.

[9] Saxena RK. A remark on a paper on M-series. Fract. Calc. Appl. Anal. 2009; 12(1): 109-10.

[10] Chouhan A, Saraswat S. Certain properties of fractional calculus operators associated with M-series. Scientia: Series A: Mathematical Sciences. 2012; 22: 25-30.

[11] Singh DK. Fractional Calculus Operators Involving Generalized M-Series. Jour. of Frac. Calc. and Appl. 2014; 5(2): 78-83.

[12] Sachan DS, Jaloree S., Integral Transforms of Generalized M-Series. Journal of Fractional Calculus and Applications. 2021; 12(1): 213-22. 